\title{
A New Inviscid Wall Boundary Condition Treatment for Embedded Boundary Cartesian Grid Schemes
}

\author{
David D. Marshall * and Stephen M. Ruffin ${ }^{\dagger}$ \\ School of Aerospace Engineering \\ Georgia Institute of Technology \\ Atlanta, GA 30332-0150
}

\begin{abstract}
This work presents a new inviscid wall boundary condition technique for embedded Cartesian grid schemes. This scheme eliminates the time step restrictions associated with the arbitrarily small control volumes that can result when the surface cuts the Cartesian control volumes. The cells adjacent to the surface are removed from the control volume formulation and are instead solved via an interpolation technique which utilizes the wall boundary conditions to build the interpolating functions. Two different interpolation techniques are presented, one without considering wall curvature and one considering wall curvature. Results are compared to a twodimensional airfoil case and a three-dimensional wing case.
\end{abstract}

\section{Nomenclature}

$C_{d} \quad$ Drag coefficient

$C_{l} \quad$ Lift coefficient

$C_{p} \quad$ Pressure coefficient

$c_{p} \quad$ Specific heat at constant pressure

CFL Courant-Friedrichs-Levy condition number

$K_{w} \quad$ Wall curvature constant combining curvatures and tangential velocity direction

$K_{\eta \xi} \quad$ Curvature of the surface along the $\xi$-direction

$K_{\eta \zeta} \quad$ Curvature of the surface along the $\zeta$-direction

$M \quad$ Mach number

$n \quad$ Normal vector from surface

$p \quad$ Pressure

$R \quad$ Gas constant

T Temperature

$\boldsymbol{u} \quad$ Velocity vector

$u \quad$ Velocity vector component

$x, y, z \quad$ Cartesian coordinate directions

$\gamma \quad$ Specific heat ratio

$\delta \quad$ Distance along surface normal

$\lambda \quad$ Velocity direction in the $\xi-\zeta$ plane

$\xi, \eta, \zeta \quad$ Geodesic coordinate system for surface

curvature formulation

\section{Subscripts}

$\xi, \eta, \zeta \quad$ Vector component in given geodesic coordinate direction

c Property at interpolation point along surface normal

$t \quad$ Vector property tangential to wall

$w \quad$ Property at wall

$0 \quad$ Stagnation properties

\footnotetext{
${ }^{*}$ Sr. Engineer, Advatech Pacifi c Inc., Edwards AFB, CA, Member AIAA

$\dagger$ Associate Professor, Member AIAA

Copyright (C) 2004 by the authors. Published by the American Institute of Aeronautics and Astronautics, Inc. with permission.
}

$9 \quad$ Surface cell centroid property in wall boundary condition development Freestream conditions

\section{Introduction}

CARTESIAN grids have been gaining popularity in the past several years because of the short grid generation times required for complex three dimensional configurations. ${ }^{1}$ This has motivated a number of researchers to develop inviscid, embedded grid Cartesian solvers that utilize the cut cell methodology to model the surfaces. $^{2-4}$

The problem of arbitrarily small cut cells was addressed as far back as Clarke et al. ${ }^{5}$ as well as Gaffney and Hassan ${ }^{6}$ in order to avoid the time stepping problems with the CFL restriction associated with these cut cells due to the small areas and volumes of these cells. The technique used by these researchers, and the predominant technique currently used, is the cell merging technique. The cell merging technique finds cut cells that are below a specified size criteria (typically below $50 \%$ of the full Cartesian cell size). These cells are then merged with neighboring full cells to create surface cells that are larger than the full Cartesian cells, see figure 1. In this case the surface cuts through a collection of cells, numbered 1-5. Cell 1 turns into a cut cell (numbered 1 in the resulting merged cells) while cells 2 and 3 are merged together into the cell numbered 2 since cell 3 is too small after the cut. Flow cell 4 remains a flow cell and cell 5 turns into a cut cell.

While the cell merging technique eliminates the extremely small cell sizes in the explicit time stepping schemes, it does not eliminate the cut cells that are above the specified threshold size. Thus the time step will still be constrained by $1 / 2$ of the full Cartesian cell size. In addition, this adds more complexity mapping the Cartesian cells to the computational control volumes which can have adverse affects on the finite vol- 


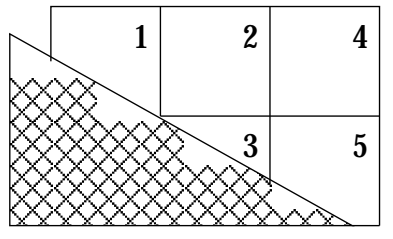

Solid surface overlayed

Cartesian Cells

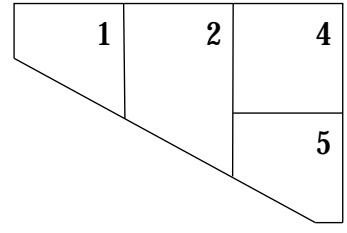

Resulting Merged

Cells
Fig. 1 Example of Merge Cell Creation

ume scheme. As an example, figure 1 shows that the treatment of the merged cell 2 results in a refinementlike interface between cell 2 and cells 4 and 5 which can cause problems for both inviscid as well as viscous cases.

This research presents an alternative treatment to the surface cells that avoids these issues. First, a brief overview of the Cartesian grid solver used for this work is presented. This is followed by a development of the pertinent details of the boundary condition development for both the flat wall assumption and the curved wall assumption. Next is the presentation of the results for the test cases which is followed by the conclusions of the work.

\section{Cartesian Solver Description}

The Cartesian grid solver used for this work is NASCART-GT, an unsteady, three-dimensional solver developed at Georgia Tech. A summary of the features will be presented here, see Marshall ${ }^{7}$ for a detailed description.

NASCART-GT is an unsteady, three-dimensional embedded grid Cartesian solver of the full Navier-Stokes or Euler equations without body forces and a perfect gas thermodynamic model. This work utilizes the inviscid solution functionality of NASCART-GT, and this description will focus on the inviscid functionality. The governing equations are solved using Roe's approximate Riemann solver coupled with a MUSCL data reconstruction technique for the inviscid fluxes. A pressure based limiter is applied to the reconstruction to dampen oscillations in high gradient regions. The overall accuracy of the scheme is as high as third order accurate in space.

The time integration is performed using a Hancock two-stage predictor-corrector scheme which is second order accurate in time. In order to accurately capture high gradient regions, a solution adaption scheme is used. The solution adaption scheme uses a combination of the velocity divergence and velocity gradient as a coarsening/refining metric.

Since no ghost-cells are used at the surface cells, the MUSCL data reconstruction limiter is used to avoid creating a computational stencil with cells that are interior to the body. This has the effect of possibly lowering the order of accuracy of the data reconstruction for some cells.
One limitation in the current version of NASCARTGT is that the size of the cells along a solid surface must be the same size. This is in order to limit the instabilities in the viscous modeling cases. ${ }^{8}$ There is no reason for this limitation to be present in the inviscid cases, and it will be removed in future versions of NASCART-GT.

\section{Wall Boundary Condition Development}

The wall boundary condition development presented here removes the surface cells from the finite volume formulation discussed above. Instead, the state for these cells is determined from an interpolation of the surrounding cells with the wall boundary conditions as additional constraints.

The formulation of the surface cell properties utilizes the state at a point normal to the surface which can be based on the surrounding cells, see figure 2 . The state at point ' $c$ ' is constructed by using a distance weighted interpolation of the of the surrounding cells (in this case cells ' 1 ' through '9'). The distance weighted interpolation places an additional restriction on the cells surrounding the surface cell. All of the cells neighboring the reference cell and the reference cell itself must be at the same refinement level as the surface cell.

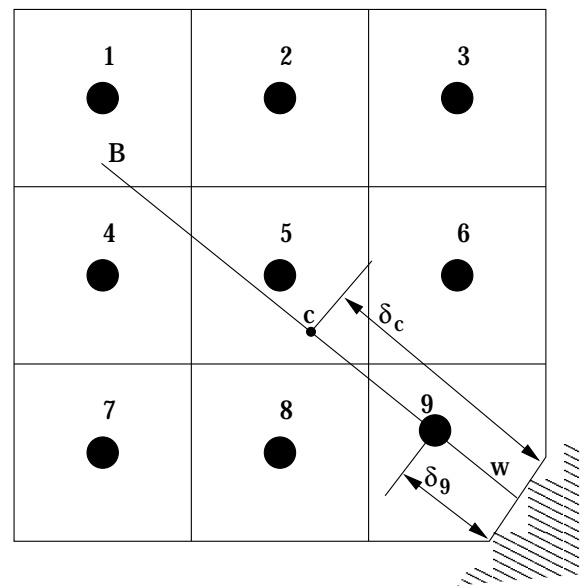

Fig. 2 Example Confi guration for Solid Boundary Treatment

Using the state at point ' $c$ ', the state at the centroid of the surface cell, labeled '9', (or the wall location, labeled ' $w$ ') can be developed by using one-dimensional relationships along the line $\overline{B w}$. The specifics of the state reconstruction relationship depends on whether the wall curvature is to be considered.

Flat Wall Development

The inviscid formulation is separated into two cases, one if the flow at point ' $c$ ' is subsonic and another if it is supersonic.

Subsonic Case The surface cell velocity is first determined by an interpolation procedure along the line $\overline{B w}$ from point ' $c$ ' to the wall utilizing the surface tangency 
wall boundary condition. The resulting relationship is

$$
\boldsymbol{u}_{9}=\boldsymbol{u}_{c}-\left(1-\frac{\delta_{9}}{\delta_{c}}\right)\left(\boldsymbol{u}_{c} \cdot \boldsymbol{n}\right) \boldsymbol{n}
$$

where $\delta_{c}$ and $\delta_{9}$ are the distances from point 'w' to points 'c' and '9', respectively. This has the effect of holding the tangential velocity constant and linearly decreasing the normal velocity to zero at the wall.

With the velocity determined at point '9', the temperature can be found by using the adiabatic relation

$$
\begin{aligned}
& T_{0}=T_{c}\left(1+\frac{\gamma-1}{2} M_{c}^{2}\right) \\
& T_{9}=T_{0}-\frac{\gamma-1}{2 c_{p}} U_{9}^{2}
\end{aligned}
$$

and the pressure can be found by using the isentropic relation

$$
p_{9}=p_{0}\left(1+\frac{\gamma-1}{2} M_{9}^{2}\right)^{\frac{\gamma}{1-\gamma}}
$$

This has the effect of correcting the thermodynamic properties for the velocity changes associated with the wall conditions.

Supersonic Case The supersonic case is split into two separate cases, one if the wall angle produces a shock and the other if it produces an expansion (or is parallel to the flow). If the wall produces a shock due to a positive wall angle, then the following standard oblique shock relations are used, see Anderson ${ }^{9}$ for the derivations

$$
\begin{aligned}
M_{n, c} & =M_{c} \sin \beta \\
\rho_{9} & =\rho_{c} \frac{(\gamma+1) M_{n, c}^{2}}{(\gamma-1) M_{n, c}^{2}+2} \\
p_{9} & =p_{c}\left[1+\frac{2 \gamma}{\gamma+1}\left(M_{n, c}^{2}-1\right)\right] \\
M_{n, 9}^{2} & =\frac{M_{n, c}^{2}+\frac{2}{\gamma-1}}{\gamma-1} M_{n, c}^{2}-1 \\
T_{n, 9} & =T_{c} \frac{p_{9}}{p_{c}} \frac{\rho_{c}}{\rho_{9}} \\
M_{9} & =M_{n, 9} \csc (\beta-\theta) \\
\tan \theta & =2 \cot \beta\left[\frac{M_{c}^{2} \sin ^{2} \beta-1}{M_{c}^{2}(\gamma+\cos 2 \beta)+2}\right]
\end{aligned}
$$

where $\beta$ is the oblique shock angle and $\theta$ is the wall angle. One additional correction is to the velocity magnitude at '9'. The subsonic formulation from above is is used to calculate the velocity direction at '9', and the velocity magnitude from the oblique shock relations is used for the final velocity magnitude.

If the wall angle produces an expansion (or is parallel to the flow) then the same subsonic velocity relations are used to calculate the velocity vector. To calculate the thermodynamic properties, the standard Busemann surface pressure coefficient relation, see Berten $^{10}$ for details, is used to determine the pressure by

$$
\begin{aligned}
C_{p} & =\frac{2}{\sqrt{M_{c}^{2}-1}} \theta+\frac{(\gamma+1) M_{c}^{2}-4 M_{c}^{2}+4}{2\left(M_{c}^{2}-1\right)^{2}} \theta^{2} \\
p_{9} & =p_{c}+\frac{\rho_{c} U_{c}^{2}}{2} C_{p}
\end{aligned}
$$

where again $\theta$ is the wall angle. From the isentropic relations, the temperature at '9' is calculated from

$$
\begin{aligned}
M_{9}^{2} & =\frac{2}{\gamma-1}\left[\left(\frac{p_{0}}{p_{9}}\right)^{\frac{\gamma-1}{\gamma}}-1\right] \\
T_{9} & =\frac{U_{9}^{2}}{\gamma R M_{9}^{2}}
\end{aligned}
$$

Curved Wall Development

While the basic model does address the time step limitation associated with the cut cell sizes mentioned above, some deficiencies of the basic model have been addressed with the curved wall model. Specifically, utilizing the surface curvature to ease the grid refinement criteria around regions of high curvature, and utilizing the governing equations to develop the interpolation relationships. The surface curvature modification requires the governing equations to be transformed into geodesic coordinates in order to incorporate the surface curvature terms. See Marshall ${ }^{7}$ for the derivation details associated with the Euler equations in both twoand three-dimensions for geodesic coordinates. The net result of this treatment is that the surface curvature is needed along the two coordinates along the surface ( $\xi$ and $\zeta$ ). The curvature of the surface along the $\xi$ direction is denoted as $K_{\eta \xi}$ and along the $\zeta$-direction is denoted as $K_{\eta \zeta}$. See figure 3 for an example surface.

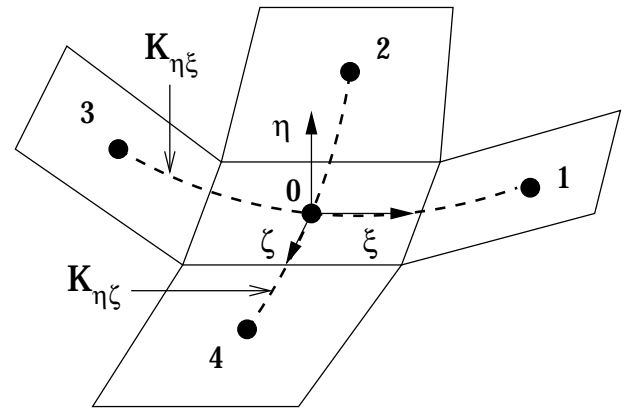

Fig. 3 Example Surface for Curvature Calculation

The normal momentum equation in the geodesic formulation is

$$
\begin{aligned}
\frac{\partial p}{\partial \eta} & =\rho\left[K_{\eta \xi} u_{\xi}^{2}+K_{\eta \zeta} u_{\zeta}^{2}\right] \\
& -\rho\left[\frac{\partial u_{\eta}}{\partial t}+\left(\frac{1}{h_{\xi}}\right) u_{\xi} \frac{\partial u_{\eta}}{\partial \xi}+u_{\eta} \frac{\partial u_{\eta}}{\partial \eta}+\left(\frac{1}{h_{\zeta}}\right) u_{\zeta} \frac{\partial u_{\eta}}{\partial \zeta}\right]
\end{aligned}
$$

where the $h$ terms are the differential length elements associated with the geodesic coordinate formulation. 
Applying equation (7) to the surface and utilizing the boundary conditions for the Euler flows (i.e. $u_{\eta}=0$, $\frac{\partial u_{\eta}}{\partial t}=0, \frac{\partial u_{\eta}}{\partial \xi}=0$ and $\left.\frac{\partial u_{\eta}}{\partial \zeta}=0\right)$ yields

$$
\frac{\partial p}{\partial \eta}=\rho\left[K_{\eta \xi} u_{\xi}^{2}+K_{\eta \zeta} u_{\zeta}^{2}\right]
$$

As with the flat wall boundary conditions, the inviscid formulation is separated into two cases, one if the flow at point ' $c$ ' is subsonic and another if it is supersonic.

Subsonic Case The surface cell state calculation starts with the assumption that the normal velocity decreases linearly and that the magnitude of the velocity does not change between points 'c' and '9'. Further, it is assumed that the tangential velocity vector does not change directions with respect to the surface coordinates between points 'c' and '9'. The following expresses these criteria

$$
\begin{aligned}
\tan \lambda_{c} & =\frac{u_{\zeta, c}}{u_{\xi, c}} \\
u_{\eta, 9} & =\frac{\delta_{9}}{\delta_{c}} u_{\eta, c} \\
u_{t, 9} & =\sqrt{U_{c}^{2}-u_{\eta, 9}^{2}} \\
u_{\xi, 9} & =u_{t, 9} \cos \lambda_{c} \\
u_{\zeta, 9} & =u_{t, 9} \sin \lambda_{c}
\end{aligned}
$$

where $u_{\xi}, u_{\eta}$ and $u_{\zeta}$ are the velocity components in the geodesic coordinate directions, $u_{t}$ is the tangential velocity and $\lambda$ is the angle made by the tangential velocity and the $\xi$-direction.

To develop the temperature relation, the adiabatic condition is used to get the following

$$
\begin{aligned}
& T_{0}=T_{c}\left(1+\frac{\gamma-1}{2} M_{c}^{2}\right) \\
& T_{9}=T_{0}-\frac{\gamma-1}{2 c_{p}} U_{9}^{2}
\end{aligned}
$$

Notice that this has the effect of holding the temperature constant since the velocity magnitudes are the same between points 'c' and '9', thus $T_{w}$ would also be equal to $T_{c}$.

With the temperature and velocity determined, the pressure relation can be developed by assuming a linear profile for the pressure curve along $\overline{B w}$ and using equation (8) for the slope of the pressure curve at the wall. To start, the equation for the pressure curve can be found to be

$$
\begin{aligned}
p & =p_{w}+\left.\delta \frac{\partial p}{\partial \eta}\right|_{w} \\
& =p_{w}\left[1+\frac{U_{w}^{2}}{R T_{w}}\left(K_{\eta \xi} \cos ^{2} \lambda_{c}+K_{\eta \zeta} \sin ^{2} \lambda_{c}\right) \delta\right]
\end{aligned}
$$

where $p$ is the pressure at a point $\delta$ distance away from the wall along $\overline{B w}$. Since the conditions at 'c' as well as the temperature and velocity at the wall are known, the wall pressure can be solved to get

$$
\begin{aligned}
p_{w} & =\frac{R T_{w}}{R T_{w}+U_{w}^{2} K_{w} \delta c} p_{c} \\
K_{w} & =K_{\eta \xi} \cos ^{2} \lambda_{c}+K_{\eta \zeta} \sin ^{2} \lambda_{c}
\end{aligned}
$$

where $K_{w}$ is the combined curvature effects in the $\xi$ - and $\zeta$-directions. With the wall pressure found, the pressure at' '9' can be found to be

$$
p_{9}=p_{w}\left(1+\frac{U_{w}^{2}}{R T_{w}} K_{w} \delta_{9}\right)
$$

and the boundary condition development is complete.

Supersonic Case The supersonic case is again split into two separate cases, one for a shock and the other for an expansion (or parallel flow). The shock case uses the oblique shock relations developed above to determine the velocity direction and thermodynamic conditions and the subsonic relations are used to determine the velocity direction. For the expansion or parallel flow case, the Busemann relations from above are used to determine the thermodynamic quantities while the subsonic relations are used to determine the velocity components.

\section{Results}

The following cases demonstrate the effectiveness of the new inviscid wall boundary conditions presented above. The first case is a transonic NACA-0012 airfoil flow. This is followed by a transonic ONERA M6 wing flow.

\section{NACA-0012 Airfoil}

This test case is a NACA-0012 airfoil in a $M_{\infty}=0.85$ flow at an angle-of-attack of $\alpha=1.00^{\circ}$. The computational boundaries are approximately 10 chords around the airfoil. Solutions are presented on a computational domain with a root grid dimension of $88 \times 80$ and 6 levels of refinement. In addition, solution adaption is performed every 200 iterations starting after 1000 iterations. Both solutions converged in approximately 20,000 iterations using local time-stepping. The final grids for the flat wall solution consists of 13,384 cells and 13,321 cells for the curved wall solution. Also, a curvature maximum of $\mathbf{4 0 . 0}$ is imposed in order to limit the large pressure gradients that can result near the leading edge. Figure 4 shows the final grid for the curved wall solution. Notice that the solution adaption has refined cells near the leading edge where the flow is going through rapid accelerations and near the shocks. The results from this case are compared with the AGARD Advisory Report results ${ }^{11}$ which presents general results from several researchers as well as detailed results for a $320 \times 64(20,480)$ cell structured grid solution.

Figures 5 and 6 show the surface pressure coefficient comparison between the NASCART-GT solutions and 


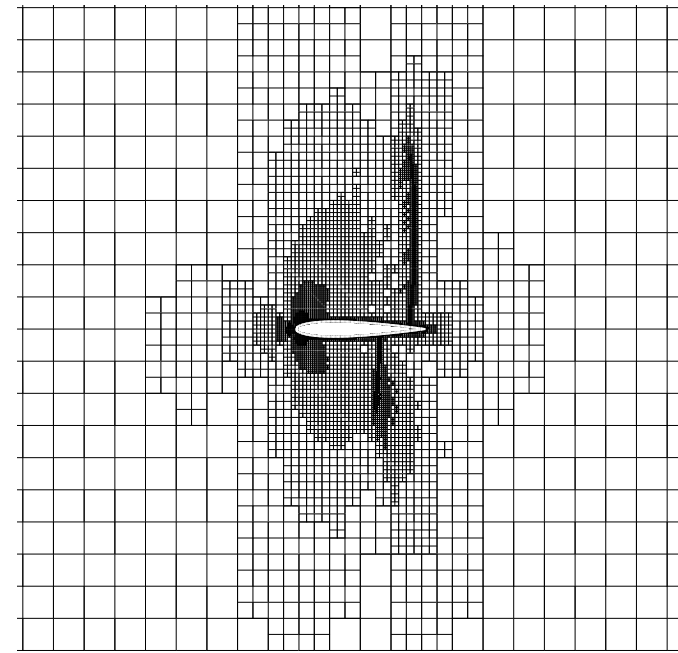

Fig. 4 Final Computational Domain for NACA-0012 Flow

the AGARD solution for the upper and lower surfaces, respectively. The curved wall solution does a better job of capturing the rapid accelerations with only slight differences at the leading edge. The upper surface shock locations are missed by approximately 0.025 chords aft and 0.035 chords aft for the curved wall and flat wall solutions respectively. For the lower surface the curved wall solution is slightly fore of the reference data, while the flat wall solution is slightly aft.

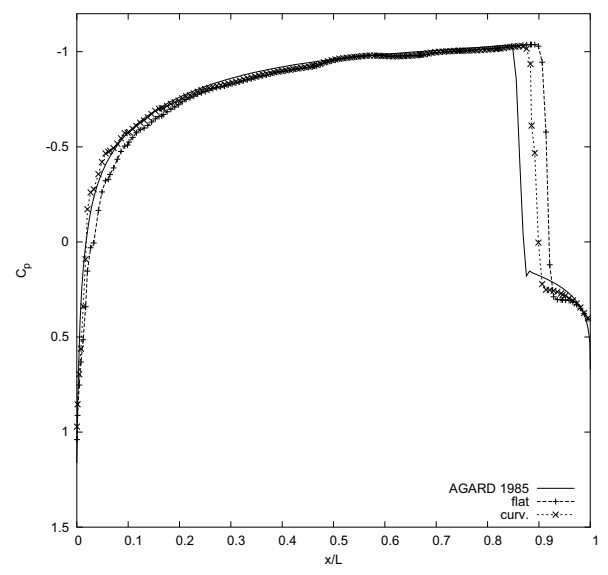

Fig. 5 NACA-0012 Upper Surface Pressure Coeffi cient

Figures 7 and 8 show the Mach contours for the flat wall and curved wall solutions, respectively. Figure 9 shows the Mach contours from the AGARD reference. All three figures use a $\Delta M=0.05$ for the contours. Both wall boundary conditions do an excellent job of capturing the flow features throughout the computational domain.

Finally, table 1 shows the lift and drag coefficients for the flat wall and curved wall cases as well as the AGARD committee results. In addition, the scatter associated with the various computed results by the AGARD researchers is also provided. The flat wall

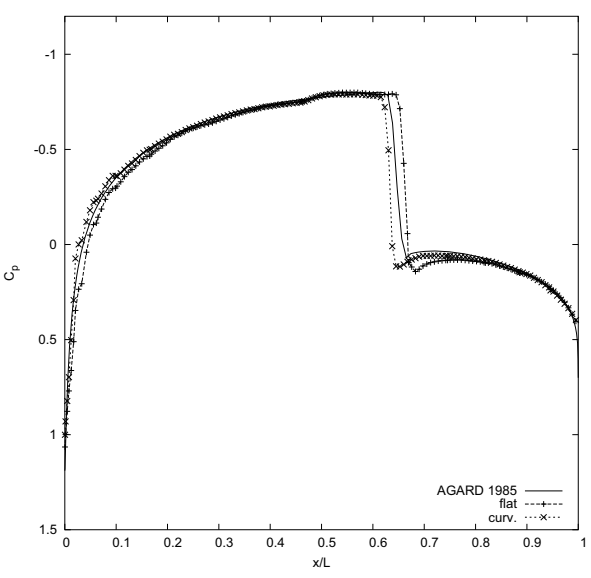

Fig. 6 NACA-0012 Lower Surface Pressure Coeffi cient

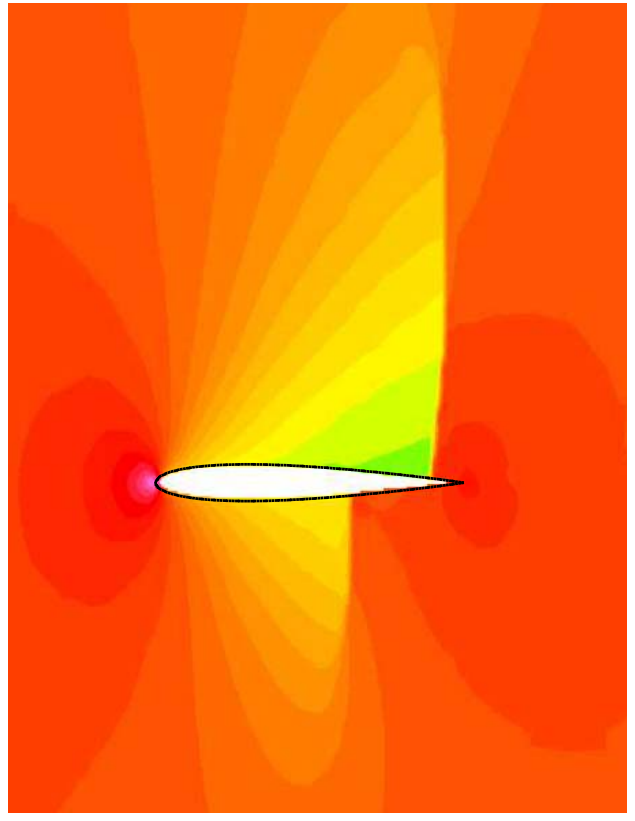

Fig. 7 Mach Contours for NACA-0012 Flat Wall

boundary condition solution performs slightly better than the curved wall boundary condition solution for the lift coefficient with a $10.9 \%$ over-prediction versus $11.8 \%$ for the flat wall solution, with each result within the scatter of the AGARD data. The curved wall boundary condition does a much better job at predicting the drag coefficient and is over the AGARD data by $; 0.1 \%$. However, the flat wall boundary condition over-predicts the drag by $34 \%$, but is close to the AGARD range. This is due to the inability of the flat wall to capture the leading edge suction peaks.

\begin{tabular}{cccc}
\hline & flat wall & curved wall & AGARD $^{11}$ (scatter) \\
\hline$C_{l}$ & 0.4007 & 0.3974 & $0.3584(0.0589)$ \\
$C_{d}$ & 0.07748 & 0.05855 & $0.0580(0.0126)$ \\
\hline
\end{tabular}

Table 1 NACA-0012 Lift and Drag Results 


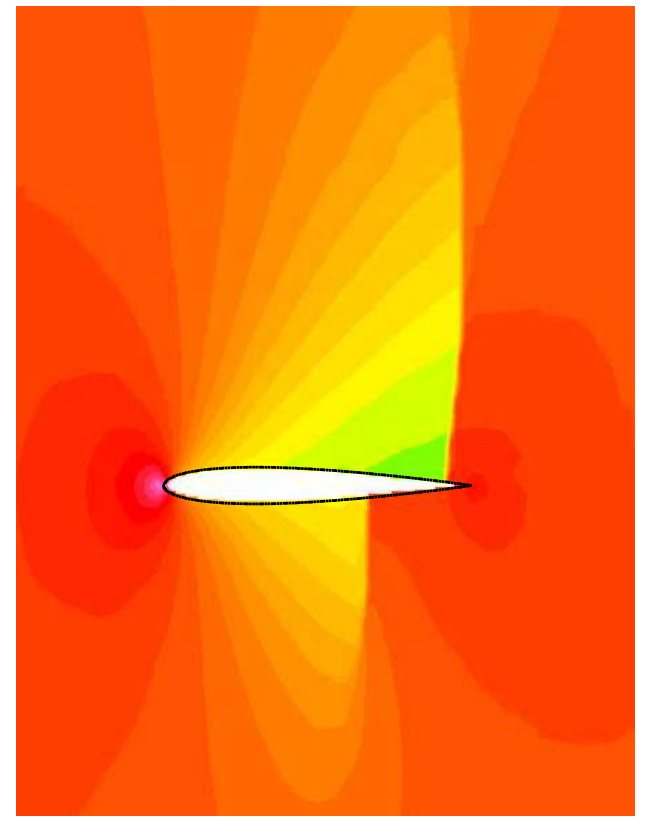

Fig. 8 Mach Contours for NACA-0012 Flow Curved Wall

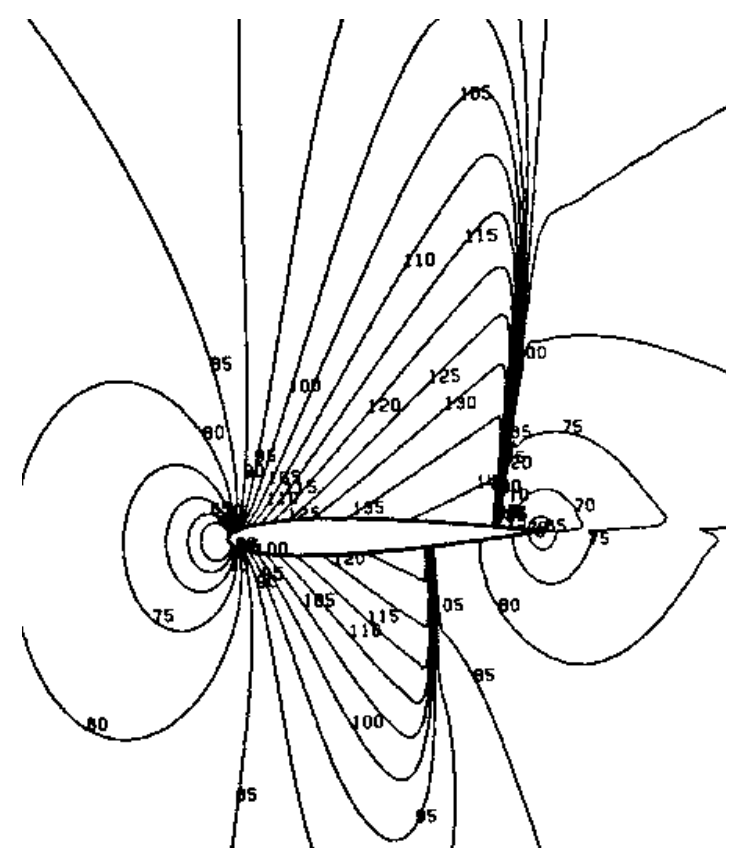

Fig. 9 NACA-0012 Mach Contours from AGARD-AR-211 ${ }^{11}$

\section{ONERA M6 Wing}

This test case is an inviscid flow around an ONERA M6 wing in a $M_{\infty}=0.84$ flow at an angle-of-attack of $\alpha=3.06^{\circ}$. The computational boundaries are 4 root chord lengths away in the $x$-, $y$ - and z-directions. The solution is presented on a computational domain with a root grid dimension of $21 \times 18 \times 22$ and 8 levels of refinement. In addition, solution adaption is performed every 200 iterations starting after 1000 iterations until 5000 iterations. The solution converged in approximately 8500 iterations. The final grid for this case consists of ap- proximately 270,000 cells with 20,357 surface cells. For this case, the flat wall boundary condition is used. Figure 10 shows the final grid for this case.

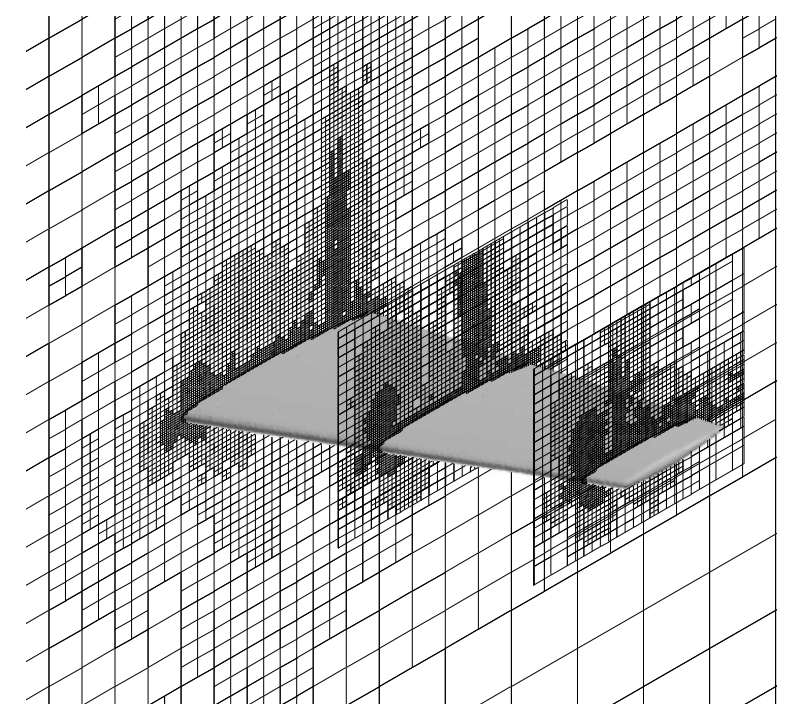

Fig. 10 Final Computational Domain for ONERA M6 Flow

The results from this case are compared with the results from AGARD Advisory Report (AGARD-AR-138) results $^{12}$ and AGARD Advisory Report (AGARD-AR211) results. ${ }^{11}$ The AGARD-AR-138 data is experimental data performed for a very high Reynolds number, $11.72 \times 10^{6}$, in order to minimize the displacement thickness effects caused by the boundary layer. The AGARDAR-211 data is a collection of computational results from several researchers for an inviscid solution to this problem.

A direction comparison of the NASCART-GT results with other inviscid solutions is difficult because other solution techniques are not limited to a single cell size throughout the entire solid surface as is NASCARTGT. However, other inviscid solutions also predict the stronger shock location aft of the experimental location, for example Aftosmis et al. ${ }^{13}$ as well as the AGARDAR-211 computational results. Both having generally good agreement with the NASCART-GT locations.

Figure 11 shows the surface pressure values at several span-wise locations for the NASCART-GT solution and the AGARD-AR-138 results. The leading edge suction peak is reasonably well captured, with some error introduced near the tip. As is typical in inviscid solutions, ${ }^{13}$ the upper surface shock locations are slightly aft of the experimental results due to the fact that the boundary layer effects are being neglected. For the inboard sections, figures 11a through 11d, there are two separate shocks on the upper surface that are present in the experimental results as well as in these results. However, the leading shock location is slightly aft of the expected location along the span-wise direction, causing the two shocks to merge closer inboard of the wing compared to the AGARD data. The lower surface shows nice agree- 
ment throughout all of the figures.

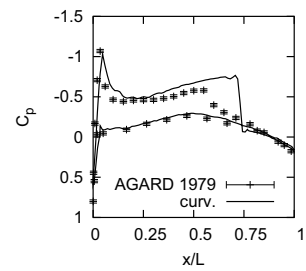

a) $z / L=0.2$

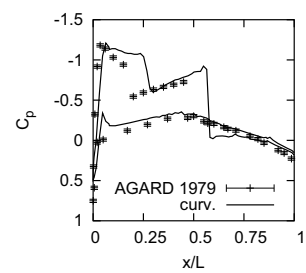

c) $z / L=0.65$

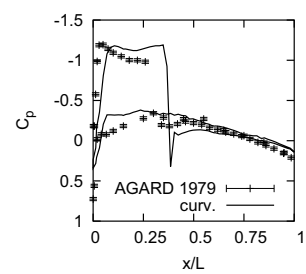

e) $z / L=0.9$

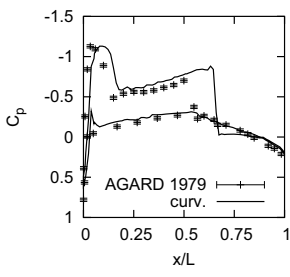

b) $z / L=0.44$

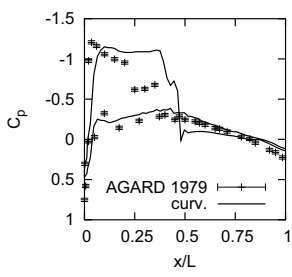

d) $z / L=0.8$

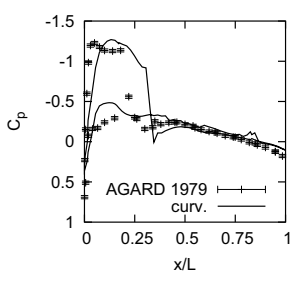

f) $z / L=0.95$
Fig. 11 Span-wise ONERA M6 Surface Pressure Coeffi cients

Figures 12 and 13 show the Mach contours on the upper surface of the wing for NASCART-GT and the AGARD-AR-211 results, respectively. Both figures use a $\Delta M=0.05$ for the contours. In these figures it is apparent that there is a lambda-shock structure on the upper surface with NASCART-GT capturing both shocks. Again, it is clear that the two socks merge further inboard.

Figures 14 and 15 show the Mach contours on the lower surface of the wing for NASCART-GT and the AGARD-AR-211 results, respectively. Both figures use a $\Delta M=0.05$ for the contours. Here there is nice agreement between the two results with only slight differences near the root and tip sections.

\section{Conclusions}

This paper presented an alternative treatment for the inviscid wall boundary condition for the cut cells in an embedded boundary Cartesian solver. This new treatment eliminates the CFL time step limitation associated with the traditional cut cell treatments by removing these cells from the finite volume formulation. This time step improvement has been observed to decrease the number of time steps to reach steady state by 4 to 5 times. While this boundary condition treatment removes the formal conservative nature of the solver for these surface cells, preliminary experiments show that the non-conservativeness is very minimal. ${ }^{7}$

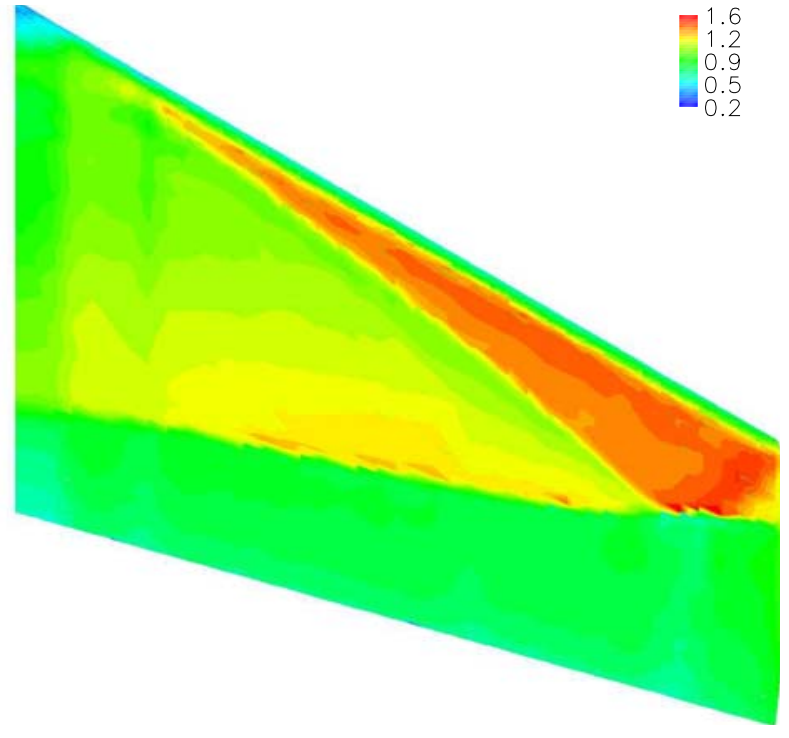

Fig. 12 ONERA M6 Upper Surface Mach Contours

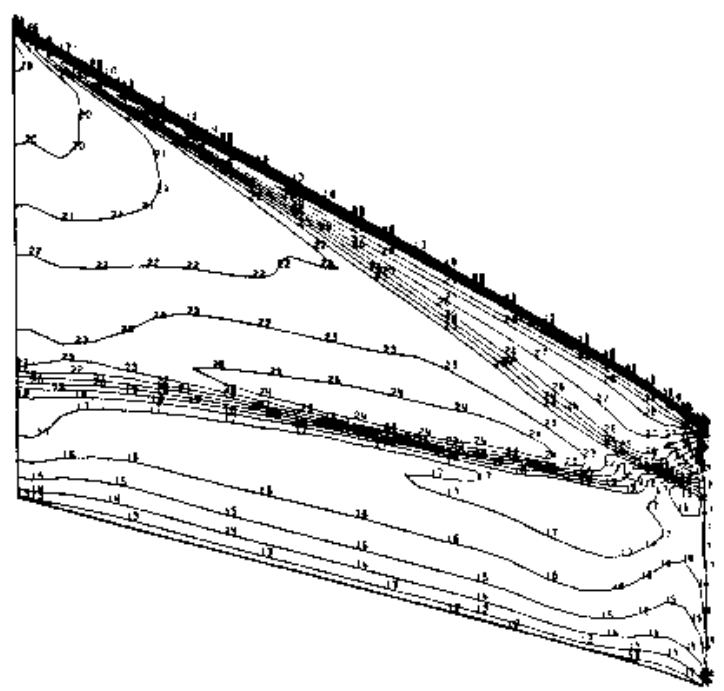

Fig. 13 ONERA M6 Upper Surface Mach Contours from AGARD-AR-211 11

\section{Acknowledgements}

David D. Marshall was supported by NASA's Graduate Student Research Program.

\section{References}

${ }^{1}$ Melton, J. E., Enomoto, F. Y., and Berger, M. J., "3D Automatic Cartesian Grid Generation for Euler Flows," 11th AIAA Computational Fluid Dynamics Conference, Orlando, FL, July 1993, AIAA-93-3386-CP.

${ }^{2}$ Berger, M. J. and LeVeque, R. J., "An Adaptive Cartesian Mesh Algorithm for the Euler Equations in Arbitrary Geometries," 9th AIAA Computational Fluid Dynamics Conference, Buffalo, NY, June 1989, AIAA-89-1930-CP.

${ }^{3}$ Melton, J. E., Berger, M. J., Aftosmis, M. J., and Wong, M. D., “3D Applications of a Cartesian Grid Euler Method," 33rd Aerospace Sciences Meeting and Exhibit, AIAA, Reno, NV, Jan. 1995, AIAA-95-0853.

${ }^{4}$ Aftosmis, M. J., Berger, M. J., and Adomavicius, G., "A Parallel 


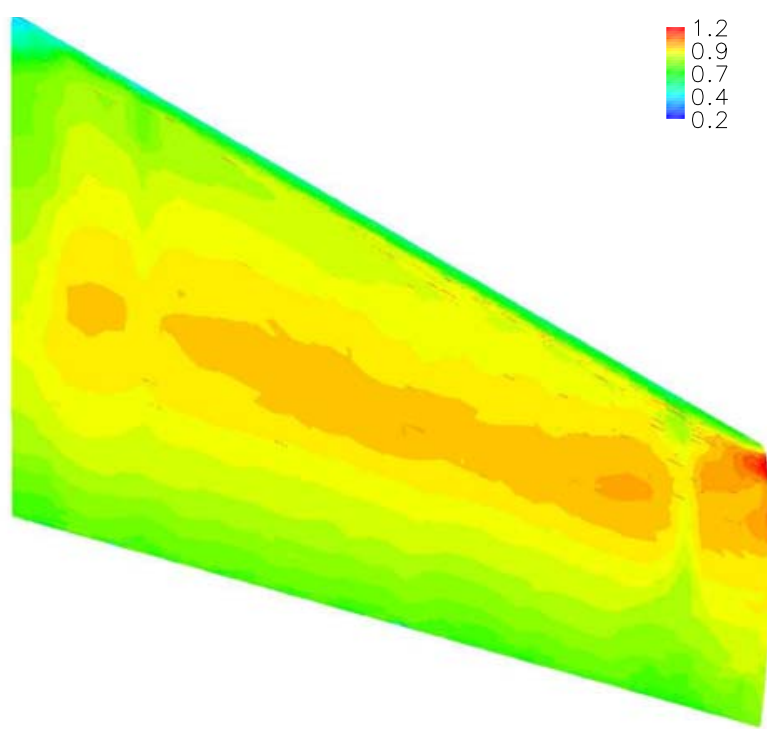

Fig. 14 ONERA M6 Lower Surface Mach Contours sian Grid Scheme for Viscous Flows using a New Viscous Wall Boundary Condition Treatment," 42nd Aerospace Sciences Meeting and Exhibit, AIAA, Reno, NV, Jan. 2004, AIAA-2004-0581.

${ }^{9}$ Anderson, Jr., J. D., Modern Compressible Flow with Historical Perspective, McGraw-Hill, Inc., New York, 2nd ed., 1990.

${ }^{10}$ Berten, J. J. and Smith, M. L., Aerodynamics for Engineers, Prentice Hall, Inc., Englewood Cliffs, NJ, 1989.

${ }^{11}$ North Atlantic Treaty Organization, "Test Cases for Inviscid Flow Field Methods," Tech. Rep. AGARD-AR-211, North Atlantic Treaty Organization Advisory Group for Aerospace Research and Development, 1985, Report of Fluid Dynamics Panel Working Group 07.

${ }^{12}$ Schmitt, V. and Charpin, F., "Pressure Distributions on the ONERAM6-Wing at Transonic Mach Numbers," Experimental Data Base for Computer Program Assessment, North Atlantic Treaty Organization, May 1979, AGARD Advisory Report 138.

${ }^{13}$ Aftosmis, M. J., Berger, M. J., and Adomavicius, G., "A Parallel Cartesian Approach for External Aerodynamics of Vehicles with Complex Geometry," Thermal and Fluids Analysis Workshop, Sept. 1999.

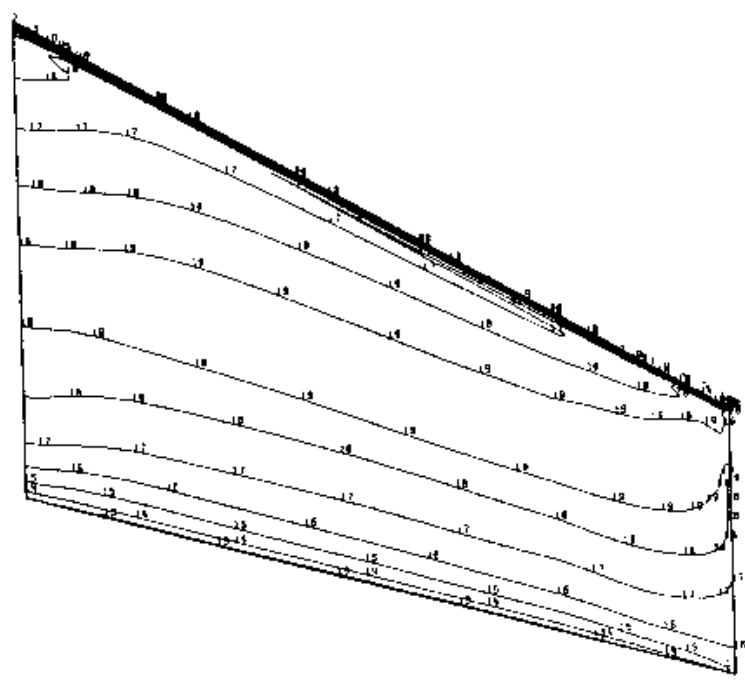

Fig. 15 ONERA M6 Lower Surface Mach Contours from AGARD-AR-211 11

Multilevel Method for Adaptively Refi ned Cartesian Grids with Embedded Boundaries," 38th Aerospace Sciences Meeting and Exhibit, AIAA, Reno, NV, Jan. 2000, AIAA-2000-0808.

${ }^{5}$ Clarke, D. K., Salas, M. D., and Hassan, H. A., "Euler Calculations for Multielement Airfoils Using Cartesian Grids," AIAA Journal, Vol. 24, No. 3, March 1986, pp. 353-358.

${ }^{6}$ Gaffney, R. L., Hassan, H. A., and Salas, M. D., "Euler Calculations for Wings Using Cartesian Grids," AIAA 25th Aerospace Sciences Meeting, Reno, NV, Jan. 1987, AIAA-87-0356.

${ }^{7}$ Marshall, D. D., Extending the Functionalities of Cartesian Grid Solvers: Viscous Effects Modeling and MPI Parallelization, Ph.D. thesis, Georgia Institute of Technology, Atlanta, GA, 2002.

${ }^{8}$ Marshall, D. D. and Ruffi n, S. M., "An Embedded Boundary Carte- 